\title{
Intrinsic and extrinsic defects in a family of coal-derived graphene quantum dots
}

\author{
Srinivasa Rao Singamaneni, ${ }^{1,2, a)}$ Johan van Tol, ${ }^{3}$ Ruquan Ye, ${ }^{4}$ and James M. Tour ${ }^{4,5,6, a)}$ \\ ${ }^{1}$ Materials Science Division, Army Research Office, Research Triangle Park, North Carolina 27709, USA \\ ${ }^{2}$ Department of Material Science and Engineering, North Carolina State University, Raleigh, North Carolina \\ 27695, USA \\ ${ }^{3}$ National High Magnetic Field Laboratory, Florida State University, 1800 E. Paul Dirac Drive, Tallahassee, \\ Florida 32310, USA \\ ${ }^{4}$ Department of Chemistry, Rice University, MS-222, 6100 Main Street, Houston, Texas 77005, USA \\ ${ }^{5}$ Department of Materials Science and NanoEngineering, Rice University, MS-222, 6100 Main Street, \\ Houston, Texas 77005, USA \\ ${ }^{6}$ Smalley Institute for Nanoscale Science and Technology, Rice University, MS-222, 6100 Main Street, \\ Houston, Texas 77005, USA
}

(Received 29 June 2015; accepted 9 November 2015; published online 23 November 2015)

In this letter, we report on the high frequency (239.2 and $336 \mathrm{GHz}$ ) electron spin resonance (ESR) studies performed on graphene quantum dots (GQDs), prepared through a wet chemistry route from three types of coal: (a) bituminous, (b) anthracite, and (c) coke; and from non-coal derived GQDs. The microwave frequency-, power-, and temperature-dependent ESR spectra coupled with computer-aided simulations reveal four distinct magnetic defect centers. In bituminous- and anthracite-derived GQDs, we have identified two of them as intrinsic carbon-centered magnetic defect centers (a broad signal of peak to peak width $=697\left(10^{-4} \mathrm{~T}\right), \mathrm{g}=2.0023$; and a narrow signal of peak to peak width $\left.=60\left(10^{-4} \mathrm{~T}\right), \mathrm{g}=2.003\right)$. The third defect center is $\mathrm{Mn}^{2+}\left({ }^{6} \mathrm{~S}_{5 / 2}, 3 \mathrm{~d}^{5}\right)$ (signal width $=61\left(10^{-4} \mathrm{~T}\right), \mathrm{g}=2.0023, \mathrm{~A}_{\text {iso }}=93\left(10^{-4} \mathrm{~T}\right)$ ), and the fourth defect is identified as $\mathrm{Cu}^{2+}\left({ }^{2} \mathrm{D}_{5 / 2}, 3 \mathrm{~d}^{9}\right)\left(\mathrm{g}_{\perp}=2.048\right.$ and $\left.\mathrm{g}_{\|}=2.279\right)$, previously undetected. Coke-derived and non-coal derived GQDs show $\mathrm{Mn}^{2+}$ and two-carbon related signals, and no $\mathrm{Cu}^{2+}$ signal. The extrinsic impurities most likely originate from the starting coal. Furthermore, Raman, photoluminescence, and ESR measurements detected no noticeable changes in the properties of the bituminous GQDs after one year. This study highlights the importance of employing high frequency ESR spectroscopy in identifying the (magnetic) defects, which are roadblocks for spin relaxation times of graphene-based materials. These defects would not have been possible to probe by other spin transport measurements. ㅇ 2015 AIP Publishing LLC. [http://dx.doi.org/10.1063/1.4936204]

Graphene quantum dots (GQDs) have unique properties $^{1-3}$ due to the quantum confinement effect, leading to potential applications in light emitting diodes, solar cells, and bio-imaging. Recent advances ${ }^{4,5}$ in chemical routes enable researchers to prepare gram-scale high quality GQDs. Of particular importance, in the recent past, GQDs have been considered $^{6,7}$ attractive candidates for solid-state quantum bits due to their long spin coherence times owing to weak spin orbit and hyperfine coupling. In such devices, the information is carried in the up or down state of electron spins in the form of spin polarized currents. However, recent spin transport measurements ${ }^{8}$ have shown that the spin-relaxation time is as much as 1000 -fold lower in graphene than predicted $^{9}$ which is in the range of micron to milliseconds, concluding that the primary source of electron spin relaxation is magnetic defects ${ }^{10}$ and interface contact resistance ${ }^{11}$ (between graphene and electrodes), but not the spin-orbit effect, as researchers have widely believed, raising the concerns on the utility of graphene-based materials for spintronics applications. The origin of those defects remains uncertain and unidentified, not possible to identify and explore their nature through spin transport measurements. Recent studies ${ }^{12}$ reporting on relatively high relaxation times

a)Electronic addresses: ssingam@ncsu.edu and tour@rice.edu.
(60-100 ns) measured on lithographically prepared GQDs have been encouraging. This motivated us to unravel such magnetic defects in GQDs that may help in generally addressing the unexpected short spin relaxation times in graphene-based materials.

Electron spin resonance (ESR) spectroscopy is the ideal spin-sensitive experimental technique of choice to identify point defects that contain unpaired spins. Atomic scale identification of such defects is possible by performing frequency- and temperature dependent measurements. This technique has been widely used in investigating the detrimental charge trapping centers in conventional and organic semiconductors, including carbon-based materials such as nanostructure graphite, carbon nanotubes, and graphene nanoribbons. ${ }^{13-15}$

By applying high frequency ESR spectroscopy, we have identified four distinct magnetic defect centers in bituminous- and anthracite coal-derived GQDs. A sharp (60 $\left.\left(10^{-4} \mathrm{~T}\right)\right)$ and a broad $\left(697\left(10^{-4} \mathrm{~T}\right)\right)$ ESR signal having gvalues of 2.003 and 2.0023 are attributed to localized carbon centered defects and conduction electrons, respectively. Finger print signatures of $\mathrm{Mn}^{2+}$ ion, which is found to be an extrinsic impurity, are observed. A previously unreported extrinsic $\mathrm{Cu}^{2+}$ ion is detected based on the characteristic spin Hamiltonian parameters. Coke- and non-coal-derived GQDs 
show $\mathrm{Mn}^{2+}$ and two-carbon related signals. Furthermore, our Raman, photoluminescence (PL), and ESR spectroscopic studies performed on bituminous coal-GQDs show that these dots are quite stable over a period of one year.

In this work, GQDs were prepared by one-step wet chemistry route ${ }^{4}$ using three types of coal: (a) bituminous (b) anthracite, and (c) coke. To briefly describe the process, these GQDs were obtained by sonicating each coal in a mixture of concentrated sulphuric and nitric acid, followed by heat treatment at 100 or $120^{\circ} \mathrm{C}$ for $24 \mathrm{~h}$. The microstructure of these GQDs was investigated by transmission electron microscopy (TEM). The TEM images show uniformly distributed sizes and shapes that are $2.96 \pm 0.96 \mathrm{~nm}$ in diameter. The observed hexagonal lattice in the fast Fourier transform images reveals that these dots are crystalline hexagonal structures. An atomic force microscope images reveal that their heights are $1.5-3 \mathrm{~nm}$, suggesting that there are two to four layers of graphene oxide-like structures. To show the quantum confinement effect of these dots, the optical properties of as prepared dots were measured by ultraviolet-visible spectroscopy, PL spectroscopy and time correlated singlephoton-counting spectroscopy. For PL and Raman measurements, the HORIBA JovinYvon Fluorolog 3 spectrometer and Renishaw inVia Raman Microscope were employed using $514 \mathrm{~nm}$ laser excitation at room temperature.

Multi-frequency ESR measurements were performed on bituminous-GQDs (bGQDs) as a function of temperature (6-290 K) and microwave attenuation (10-30 dB) at two distinct microwave high frequencies (239.2 and $336 \mathrm{GHz}$ ) using the quasioptical spectrometer that has been developed ${ }^{16}$ at the National High Magnetic Field Laboratory, Florida. This setup was a superheterodyne spectrometer, employing a quasi-optical submillimeter bridge and operating in reflection mode without a cavity and with a sweepable $12.5 \mathrm{~T}$ superconducting magnet. The optimized incident power on the sample was $\sim 20 \mu \mathrm{W}$.

Figure 1 presents ESR spectra collected at $290 \mathrm{~K}$ and $239.2 \mathrm{GHz}$, displayed both in absorption and dispersion modes. No background signal was detected from the empty

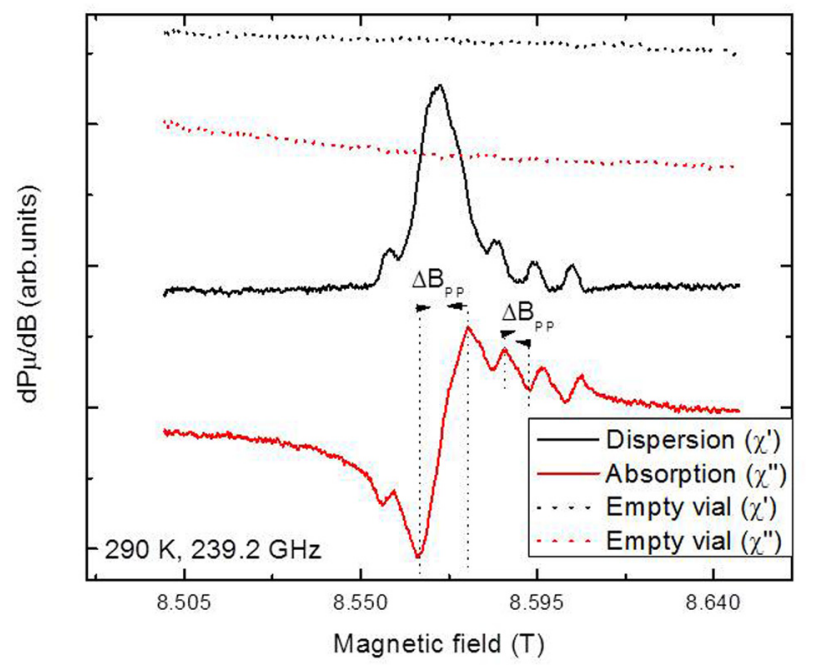

FIG. 1. ESR spectra from bGQDs collected at $239.2 \mathrm{GHz}$ and $290 \mathrm{~K}$ both in absorption (red continuous curve) and dispersion (black continuous curve) modes. Spectra from the empty vial (dotted lines) were used as baseline. vial (dotted lines). This plot shows at least two distinct wellresolved ESR spectra. A spectrum with the fingerprint signatures of $\mathrm{Mn}^{2+}\left({ }^{6} \mathrm{~S}_{5 / 2}, 3 \mathrm{~d}^{5}\right)$ signal and a peak-to-peak line width $\left(\Delta \mathrm{B}_{\mathrm{pp}}\right)$ of $\sim 61\left(10^{-4} \mathrm{~T}\right)$, g-factor of 2.0023 is characterized by isotropic hyperfine splitting $\left(\mathrm{A}_{\text {iso }}\right)$ of $93\left(10^{-4} \mathrm{~T}\right)$. The second spectra contain a sharp signal with a peak-topeak line width of $60\left(10^{-4} \mathrm{~T}\right)$, with the g-value close to 2.003, was attributed to carbon-related defect centers such as dangling carbon bonds. ${ }^{15,17,18}$ We find that the ESR signal shape does not show power dependence measured at 239.2 GHz, $6 \mathrm{~K}$, as shown in Fig. S1 of supplementary material. ${ }^{19}$ The spectra show very little temperature dependence ${ }^{19}$ (see Fig. S2 of the supplementary material).

The spectra were analyzed more closely by fitting the experimental data, carried out both in absorption and dispersion modes. As illustrated in Figs. 2(a) and 2(b) as a first attempt, we tried to fit the experimental spectrum (curve in black) with only two components (blue curve), comprised of the spectrum originating from the $\mathrm{Mn}^{2+}$ center and a sharp Lorentzian signal. Upon closer inspection, it was noticed that the spectral features could not be adequately reproduced. As a next step, the spectrum was fit with the addition of a broad Lorentzian as a third component. As one can see, the experimental (black curve) and simulated (red curve) spectra agree consistently well, both in absorption and dispersion modes. From the derived spin Hamiltonian parameters, we have identified these signals as: (a) an ESR signal ${ }^{20,21}$ with the peak-to-peak line width of $697\left(10^{-4} \mathrm{~T}\right)$, a g-value of 2.0023 , may originate from the mobile pi-carriers propagating in the interiors of graphene sheets; (b) a narrow signal having a peak-to-peak line width of $60\left(10^{-4} \mathrm{~T}\right)$ with the g-value of 2.003 which are intrinsic carbon-centered paramagnetic centers; ${ }^{15,17,18}$ and (c) a $\mathrm{Mn}^{2+}$ signal $^{22}$ which is an extrinsic impurity. We have also simulated the experimental spectra measured at various temperatures ${ }^{19}$ (see Fig. S3 in the supplementary material) using Zeeman and hyperfine terms in the spin Hamiltonian. The experimental spectra at all temperatures and at two different frequencies show the typical six-line pattern of $\mathrm{Mn}^{2+}$ corresponding to $\mathrm{M}_{\mathrm{s}}=\mid-1 / 2>\rightarrow$ $\mid+1 / 2>\left(\Delta \mathrm{M}_{\mathrm{s}}=1 ; \Delta \mathrm{M}_{\mathrm{I}}=0\right)$ transitions coupled to the ${ }^{55} \mathrm{Mn}$ nuclear spin, in addition to other two Lorentzian components. $\mathrm{Mn}^{2+}$ ESR spectra are found to be practically independent of temperature. This supports the conclusion that $\mathrm{Mn}^{2+}$ is not substituted in the graphene lattice, but is more likely an isolated defect. ${ }^{23}$ This is consistently reproduced both in absorption and dispersion spectra collected at $239.2 \mathrm{GHz}$.

To gain confidence and look for additional defect centers, the ESR spectra of bGQDs was measured at further high microwave frequency $336 \mathrm{GHz}$ and $3 \mathrm{~K}$. The experimental spectrum is presented in Figs. 3(a) and 3(b) (curve in black). As shown in Fig. 3(a), the high field region is fitted with two Lorentzian components together with $\mathrm{Mn}^{2+}$ spectra (shown in red), consistent with the $239.2 \mathrm{GHz}$ spectra as discussed above. The difference in spectrum is shown in green. The spin Hamiltonian parameters derived from $\mathrm{Mn}^{2+} \mathrm{ESR}$ spectra are found to be practically the same as that of 239.2 GHz spectrum. The g-values of two carbon-related Lorentzian signals remain the same as those of $239.2 \mathrm{GHz}$. However, the narrow and broad carbon-derived signals have broadened further, whose line widths are $\sim 200\left(10^{-4} \mathrm{~T}\right)$ and 

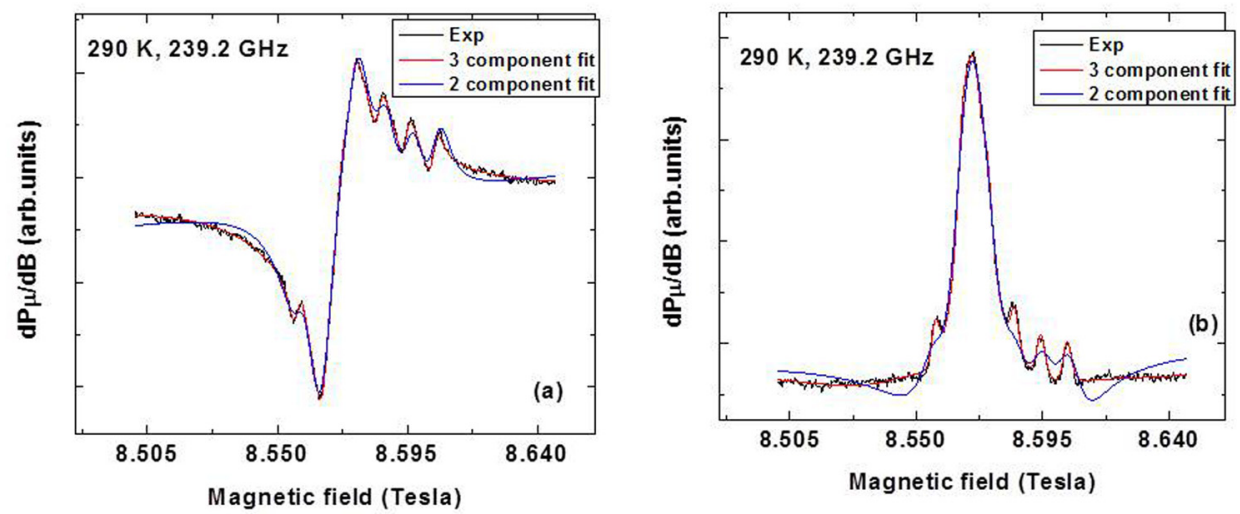

FIG. 2. Comparison of $239.2 \mathrm{GHz}$ $(290 \mathrm{~K})$ experimental (black) and simulated ESR spectra by two (blue) and three (red) components fits in (a) absorption and (b) dispersion modes. $\sim 890\left(10^{-4} \mathrm{~T}\right)$, respectively. Similar line broadening was observed from graphene nanoribbons as reported in our previous work. $^{24}$

The low field ESR spectra are shown in Fig. 3(b), which merits attention. The low field ESR spectrum was fitted using an axial spin-Hamiltonian of the form

$$
\begin{aligned}
\mathrm{H}= & \mathrm{g}_{\|} \beta \mathrm{B}_{\mathrm{z}} \mathrm{S}_{\mathrm{z}}+\mathrm{g}_{\perp} \beta(\mathrm{BxSx}+\mathrm{BySy})+\mathrm{A}_{\|} \mathrm{S}_{\mathrm{z}} \mathrm{I}_{\mathrm{z}} \\
& +\mathrm{A}_{\perp}\left(\mathrm{S}_{\mathrm{x}} \mathrm{I}_{\mathrm{x}}+\mathrm{S}_{\mathrm{y}} \mathrm{I}_{\mathrm{y}}\right) .
\end{aligned}
$$

Here " $\mathrm{z}$ " is the symmetry axis of individual $\mathrm{Cu}^{2+}$ ions, $\beta$ is the Bohr magneton, $\mathrm{A}_{\|}$and $\mathrm{A}_{\perp}$ are the parallel and perpendicular components of hyperfine tensor " $A$;" $\mathrm{g}_{\|}$and $\mathrm{g}_{\perp}$ are the parallel and perpendicular components of " $\mathrm{g}$ " tensor; $\mathrm{H}_{\mathrm{x}}$, $\mathrm{H}_{\mathrm{y}}$, and $\mathrm{H}_{\mathrm{z}}$ are the components of magnetic field; $\mathrm{S}_{\mathrm{x}}, \mathrm{S}_{\mathrm{y}}$, and $S_{z}$ are components of spin operators of the electron; and $I_{x}$, $I_{y}$, and $I_{z}$ are the spin operators of the nucleus. Upon careful examination, it is evident that these spectra exhibit resonance signals (powder pattern) similar to those reported ${ }^{25-27}$ in earlier studies on $\mathrm{Cu}^{2+}\left({ }^{2} \mathrm{D}_{5 / 2}, 3 \mathrm{~d}^{9}\right)$ signal with $\mathrm{S}=1 / 2$ and a nuclear spin $\mathrm{I}=3 / 2$ for both ${ }^{63} \mathrm{Cu}$ (natural abundance of $69 \%$ ) and ${ }^{65} \mathrm{Cu}$ (natural abundance of $31 \%$ ), and therefore $(2 \mathrm{I}+1)$, i.e., four parallel and four perpendicular lines would be expected, typically characterized by $\mathrm{g}_{\perp}=2.048$ and $\mathrm{g}_{\|}=2.279$, as derived from the computer simulations of the experimental spectrum shown in the red curve. The observed $\mathrm{g}_{\|}$and $\mathrm{g}_{\perp}$ values are characteristic of $3 \mathrm{~d}^{9}$ Jahn-Teller ions coordinated by six $\mathrm{O}^{2-}$ ligands that form an oxygen octahedron, elongated along the $\mathrm{z}$-axis. Because the perpendicular component of g-value is greater than the parallel component, which is in turn greater than the free electron g-value (2.0023), the ground state of $\mathrm{Cu}^{2+}$ ions can be inferred as $\mathrm{d}_{\mathrm{x} 2-\mathrm{y} 2}\left({ }^{2} \mathrm{~B}_{1 \mathrm{~g}}\right)$, the $\mathrm{Cu}^{2+}$ ions are present in octahedral sites with tetragonal distortion. The hyperfine components belonging to the ${ }^{63} \mathrm{Cu}$ and ${ }^{65} \mathrm{Cu}$ isotopes are not resolved in the observed EPR spectra, because their nuclear magnetic moments are closely similar (nuclear magnetic moment is $7.1 \%$ higher for ${ }^{65} \mathrm{Cu}$ than that for ${ }^{63} \mathrm{Cu}$ ). This defect was not detected in previous graphene-based materials studies, thus emphasizing the importance of high frequency ESR spectroscopy in identifying the defects that cannot be detected by other conventional spin-transport measurements. To make our hypothesis and explanation firm, the ESR measurements were repeated on anthracite-, coke-, and on non-coalderived GQDs (data not shown). In anthracite-derived GQDs, ESR detected all four magnetic defect centers, similar to the case of bituminous-derived GQDs. Coke-derived and non-coal derived GQDs show only $\mathrm{Mn}^{2+}$ and twocarbon related signals, but not $\mathrm{Cu}^{2+}$ signal.

No $\mathrm{Mn}^{2+}$ or $\mathrm{Cu}^{2+}$ containing materials were used in the preparation of the GQDs. It is probable that they were present in trace amounts in the starting material, and could not be detected using other analytical techniques, while high frequency ESR has proven to be a sophisticated probe. Based on the g-value and line width, the broad signal could arise from the conductive $\mathrm{p}$-carriers propagating in the interiors of graphene sheets, while the narrow ESR signal may stem from non-bonding localized electronic states at the edges of the sheets. From the ESR results, collected at measured frequencies and temperatures, we also infer that the proposed defect is not a di-vacancy as no forbidden ESR transition could be found, though intensely searched for. The presence of $\mathrm{Mn}^{2+}$ defects in graphene oxide produced using the Hummers method was reported by previous researchers.
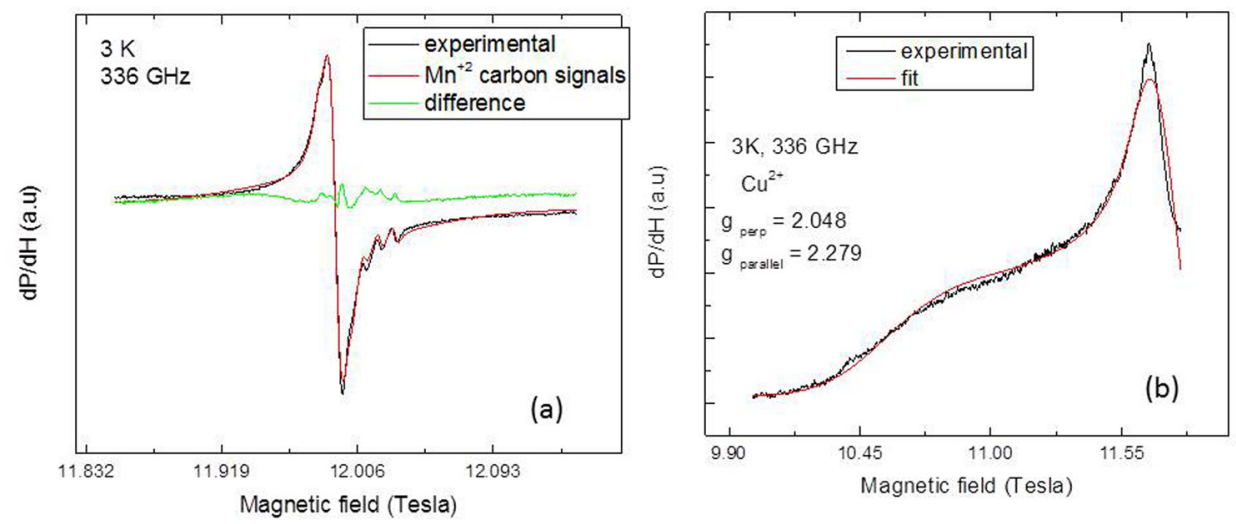

FIG. 3. $336 \mathrm{GHz}$ ESR spectra measured at $3 \mathrm{~K}$ on bGQDs shown in high field (a) and low field (b) regions. Also, the experimental (black) and fitted (red) spectra are compared in both cases in the same plots. 
These external isolated defects $\mathrm{Mn}^{2+}$ and $\mathrm{Cu}^{2+}$ may likely form paramagnetic charge-transfer complexes with the graphene planes and contribute to spin relaxation times of the bGQDs. It should be mentioned that these measurements were performed on another type of graphene quantum dots produced $^{4}$ from anthracite as a function of temperature and microwave frequency. All four magnetic centers were observed (data not shown) to be present in the graphene quantum dots.

Aging studies were carried out on the bGQDs. As depicted in Figs. 4(a)-4(c), we have measured Raman, ESR, and PL spectra of bGQDs one year after their synthesis. The Raman spectra show clear signatures of graphene-based materials $^{28,29}$ collected from pristine (red) and aged (black) samples. The Raman spectra (Fig. 4(a)) of both samples show two distinct $D$ and $G$ peaks, at $\sim 1360 \mathrm{~cm}^{-1}$ and $1600 \mathrm{~cm}^{-1}$, respectively. The $\mathrm{G}$ band corresponds to the $\mathrm{E}_{2 \mathrm{~g}}$ phonon at the Brillouin zone center. The $\mathrm{D}$ band comes from transverse optical (TO) phonons around the K-point of the Brillouin zone and requires a defect for its activation. The intensity of the $\mathrm{D}$ band is less than that of the $\mathrm{G}$ band, as expected for graphene-based materials. The 2D band (the second order of the $\mathrm{D}$ band), at $\sim 2700 \mathrm{~cm}^{-1}$, appears to be broadened similar to previous reports. ${ }^{28,30}$ This particular broadening in the $2 \mathrm{D}$ band could be due to the relaxation of the double-resonance Raman selection rules associated with the random orientation of GQDs with respect to each other. The PL spectra (see, Fig. 4(b)) of pristine (red) and aged (black) bGQDs in deionized (DI) water (pH is slightly less than 7), excited at $345 \mathrm{~nm}$, show a strong peak at $\sim 520 \mathrm{~nm}$ consistent with the previous reports attributed to surface effects and defects. ${ }^{4}$ As shown in Fig. 4(c), the $239.2 \mathrm{GHz}$ ESR spectra measured at $290 \mathrm{~K}$ of pristine (red) and aged (black) samples are practically identical. With prolonged aging during one year in an open atmosphere, no significant changes in the physical properties of the GQDs were observed through Raman, ESR, and PL spectroscopy. This evidence supports the conclusion that there is no structural reconstruction of the GQDs and that they retain their pristine characteristics. For instance, previous researchers ${ }^{13,31}$ have observed strong structural relaxation in nano graphite through Raman spectroscopy. Strong aging effects have been observed in nanostructured graphite and carbon nanofoam. Furthermore, drastic changes in the electrical properties of graphene-based transistors exposed to ambient conditions have been observed due to aging over a month. Such changes were attributed to structural reconstruction and interaction with atmospheric gases.

In summary, using spin-sensitive high frequency ESR spectroscopy, we have identified two intrinsic (broad signal: width $=697\left(10^{-4} \mathrm{~T}\right), \mathrm{g}=2.0023$; and narrow signal: width $\left.=60\left(10^{-4} \mathrm{~T}\right), \mathrm{g}=2.003\right)$ carbon-centered magnetic defect centers. The third defect center is $\mathrm{Mn}^{2+}$ (signal width $\left.=61\left(10^{-4} \mathrm{~T}\right), \mathrm{g}=2.0023, \mathrm{~A}_{\text {iso }}=93\left(10^{-4} \mathrm{~T}\right)\right)$, and the fourth defect is $\mathrm{Cu}^{2+}\left(\mathrm{g}_{\perp}=2.048\right.$ and $\left.\mathrm{g}_{\|}=2.279\right)$, which are extrinsic impurities most likely originating from the starting bituminous coal, where the $\mathrm{Cu}^{2+}$ defect was previously unreported. Furthermore, Raman, PL, and ESR measurements detected no noticeable changes in the properties of the GQDs after one year, suggesting that they are structurally stable. This study highlights the importance of employing high frequency ESR spectroscopy in identifying magnetic defects that are roadblocks for useful spin relaxation times of graphene-based materials. These defects are not possible to probe using other spin transport measurements. In addition to ripples in graphene sheets, these defects can act as strong unwanted scattering centers and limit the spin relaxation times. That might explain why there is a large discrepancy in
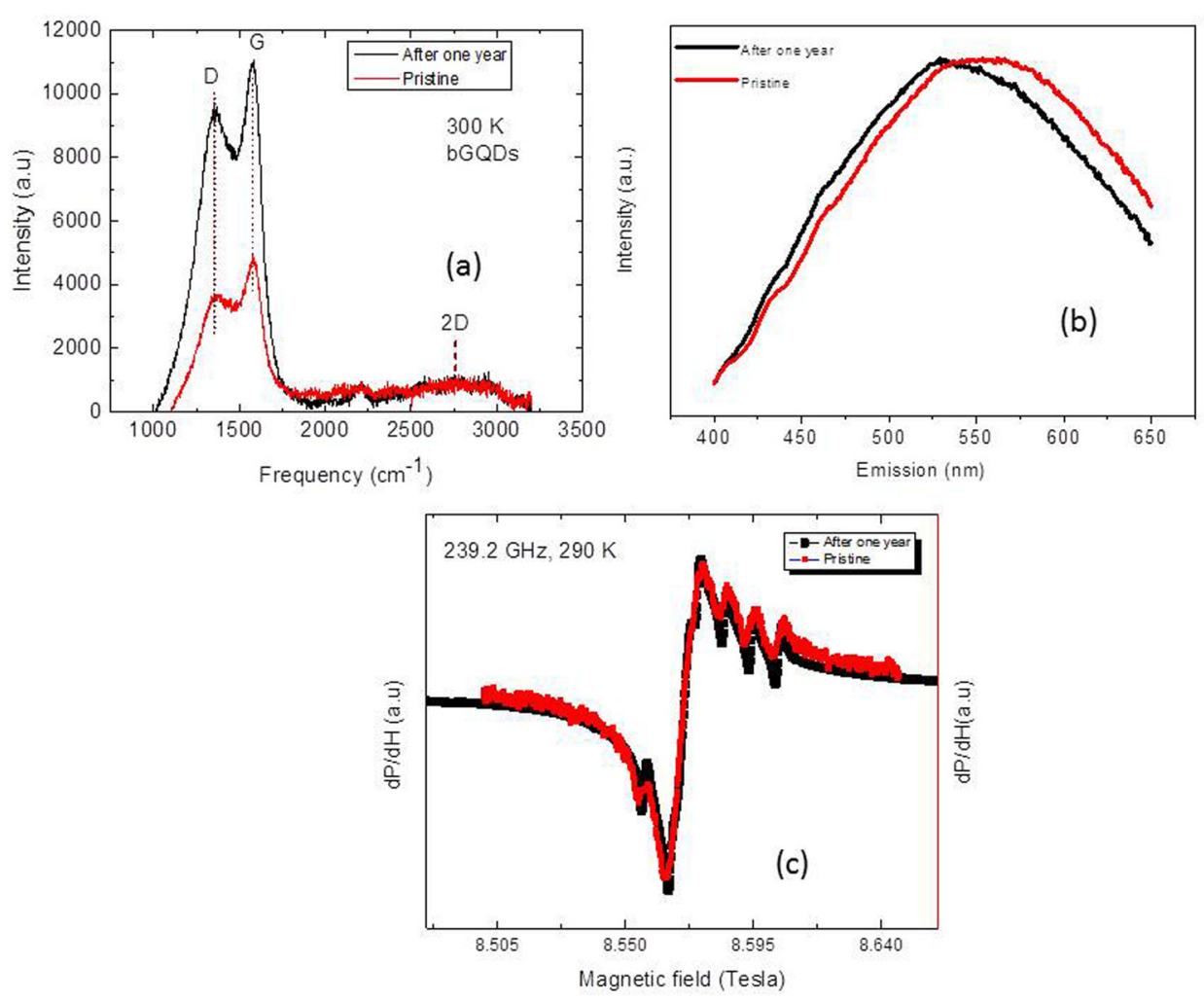

FIG. 4. Comparison of the properties of pristine (red) and aged (black) bGQDs through room temperature (a) Raman, (b) Photoluminescence (PL), and (c) ESR spectroscopic studies. 
spin relaxation times between theoretical and experimental studies, ${ }^{10}$ an on-going topic of intense investigation. The observed extrinsic magnetic impurities such as $\mathrm{Mn}^{2+}$ and $\mathrm{Cu}^{2+}$ in graphene quantum dots could have stemmed from the starting coal material. They are a strong source of spin scattering and could limit the spin life time in real graphene devices. To get theoretically predicted long spin life times and to bridge the gap between the experimental and theoretical values, which has been a puzzling question in graphene spintronics, the future research should aim to eliminate/minimize such magnetic defects even in the best quality graphene materials.

S.R.S. acknowledges NAS/NRC for postdoctoral fellowship. The National High Magnetic Field Laboratory was supported by NSF Cooperative Agreement No. DMR-1157490, and by the State of Florida. The work at Rice University was supported by the Air Force Office of Scientific Research FA9550-14-1-0111. We thank A. Metzger for synthesis of the non-coal derived GQDs. J.M.T. has interest in a company that has licensed technology on graphene quantum dot manufacture and applications. This is reported to and overseen by the Rice University Office of Research Compliance.

${ }^{1}$ L. Li, G. Wu, G. Yang, J. Peng, J. Zhao, and J.-J. Zhu, Nanoscale 5, 4015 (2013).

${ }^{2}$ S. Zhu, J. Zhang, C. Qiao, S. Tang, Y. Li, W. Yuan, B. Li, L. Tian, F. Liu, R. Hu, H. Gao, H. Wei, H. Zhang, H. Sun, and B. Yang, Chem. Commun. 47, 6858 (2011).

${ }^{3}$ V. Gupta, N. Chaudhary, R. Srivastava, G. D. Sharma, R. Bhardwaj, and S. Chand, J. Am. Chem. Soc. 133, 9960 (2011).

${ }^{4}$ R. Ye, C. Xiang, J. Lin, Z. Peng, K. Huang, Z. Yan, N. P. Cook, E. L. G. Samuel, C.-C. Hwang, G. Ruan, G. Ceriotti, A.-R. O. Raji, A. A. Martı', and J. M. Tour, Nat. Commun. 4, 2943 (2013).

${ }^{5}$ L. Wang, Y. Wang, T. Xu, H. Liao, C. Yao, Y. Liu, Z. Li, Z. Chen, D. Pan, L. Sun, and M. Wu, Nat. Commun. 5, 5357 (2014).

${ }^{6}$ B. trauzettel, D. V. Bulaev, D. Loss, and G. Burkard, Nat. Phys. 3, 192 (2007).

${ }^{7}$ M. O. Hachiya, G. Burkard, and J. C. Egues, Phys. Rev. B 89, 115427 (2014).

${ }^{8}$ N. Tombros, C. Jozsa, M. Popinciuc, H. T. Jonkman, and B. J. van Wees, Nature 448, 571 (2007).

${ }^{9}$ C. Ertler, S. Konschuh, M. Gmitra, and J. Fabian, Phys. Rev. B 80, 041405 (2009).
${ }^{10}$ M. B. Lundeberg, R. Yang, J. Renard, and J. A. Folk, Phys. Rev. Lett. 110, 156601 (2013).

${ }^{11}$ H. Idzuchi, A. Fert, and Y. Otani, Phys. Rev. B 91, 241407(R) (2015).

${ }^{12}$ C. Volk, C. Neumann, S. Kazarski, S. Fringes, S. Engels, F. Haupt, A. Muller, and C. Stampfer, Nat. Commun. 4, 1753 (2013).

${ }^{13}$ J. Kausteklis, P. Cevc, D. Ařcon, L. Nasi, D. Pontiroli, M. Mazzani, and M. Ricc', Phys. Rev. B 84, 125406 (2011).

${ }^{14}$ B. Náfrádi, N. M. Nemes, T. Fehér, L. Forro, Y. Kim, J. E. Fischer, D. E. Luzzi, F. Simon, and H. Kuzmany, Phys. Status Solidi B 243, 3106 (2006).

${ }^{15}$ S. S. Rao, A. Stesmans, K. Keunen, D. V. Kosynkin, A. Higginbotham, and J. M. Tour, Appl. Phys. Lett. 98, 083116 (2011).

${ }^{16}$ J. van Tol, L. C. Brunel, and R. J. Wylde, Rev. Sci. Instrum. 76, 074101 (2005).

${ }^{17}$ S. S. Rao, A. Stesmans, J. V. Novyen, P. Jacobs, and B. Sels, J. Phys.: Condens. Matter 23, 455801 (2011).

${ }^{18}$ S. S. Rao, A. Stesmans, D. V. Kosynkin, A. Higginbotham, and J. M. Tour, New J. Phys. 13, 113004 (2011).

${ }^{19}$ See supplementary material at http://dx.doi.org/10.1063/1.4936204 for power dependence of $239.2 \mathrm{GHz}$ ESR spectra measured at $290 \mathrm{~K}$ on bGQDs; The temperature dependence of $336 \mathrm{GHz}$ ESR spectra measured on bGQDs collected at 8, 100, and $290 \mathrm{~K}$; Experimental and computer fitted 239.2 GHz ESR spectra collected on bGQDs at 6, 10, and $290 \mathrm{~K}$.

${ }^{20}$ C. Buschhaus and E. Dormann, Phys. Rev. B 66, 195401 (2002).

${ }^{21}$ S. S. Rao, A. Stesmans, Y. Wang, and Y. Chen, Physica E 44, 1036 (2012).

${ }^{22}$ A. M. Panich, A. I. Shames, and N. A. Sergeev, Appl. Magn. Reson. 44, 107 (2013).

${ }^{23}$ A. M. Panich, A. I. Shames, A. E. Aleksenskii, and A. Dideikin, Solid State Commun. 152, 466 (2012).

${ }^{24}$ S. S. Rao, A. Stesmans, J. van Tol, D. V. Kosynkin, A. HigginbothamDuque, W. Lu, A. Sinitskii, and J. M. Tour, ACS Nano 6, 7615 (2012).

${ }^{25}$ N. J. Trappeniers, F. S. Stibbe, and J. L. Rao, Chem. Phys. Lett. 56, 10 (1978).

${ }^{26}$ B. V. Padlyak, W. Wojtowicz, V. T. Adamiv, Ya. V. Burak, and I. M. Teslyuk, Acta Phys. Pol., A 117, 122 (2010).

${ }^{27}$ R. P. S. Chakradhar, K. P. Ramesh, J. L. Rao, and J. Ramakrishna, J. Phys.: Condens. Matter 15, 1469 (2003).

${ }^{28}$ S. Kim, D. H. Shin, C. O. Kim, S. S. Kang, S. S. Joo, S.-H. Choi, S. W. Hwang, and C. Sone, Appl. Phys. Lett. 102, 053108 (2013).

${ }^{29}$ J. Peng, W. Gao, B. K. Gupta, Z. Liu, R. Romero-Aburto, L. Ge, L. Song, L. B. Alemany, X. Zhan, G. Gao, S. A. Vithayathil, B. A. Kaipparettu, A. A. Marti, T. Hayashi, J.-J. Zhu, and P. M. Ajayan, Nano Lett. 12, 844 (2012).

${ }^{30}$ L. M. Malard, M. A. Pimenta, G. Dresselhaus, and M. S. Dresselhaus, Phys. Rep. 473, 51 (2009).

${ }^{31}$ A. V. Rode, E. G. Gamaly, A. G. Christy, J. G. Fitz Gerald, S. T. Hyde, R. G. Elliman, B. Luther-Davies, A. I. Veinger, J. Androulakis, and J. Giapintzakis, Phys. Rev. B 70, 054407 (2004). 